\title{
Measuring strong one-bond dipolar couplings using REDOR in magic- angle spinning solid-state NMR
}

\section{Journal Article}

\section{Author(s):}

Jain, Mukul G.; Mote, Kaustubh R.; Hellwagner, Johannes; Rajalakshmi, G.; Ernst, Matthias (D); Madhu, P. K.; Agarwal, Vipin

Publication date:

2019

\section{Permanent link:}

https://doi.org/10.3929/ethz-b-000338133

\section{Rights / license:}

In Copyright - Non-Commercial Use Permitted

\section{Originally published in:}

The Journal of Chemical Physics 150(13), https://doi.org/10.1063/1.5088100 
Publishimgeasuring strong one-bond dipolar couplings using REDOR in magic-angle spinning solid-state NMR

Mukul G. Jain, ${ }^{1}$ Kaustubh R. Mote, ${ }^{1}$ Kaustubh R. Mote, ${ }^{1}$ Johannes Hellwagner, ${ }^{2}$ G.

Rajalakshmi, ${ }^{1}$ Matthias Ernst, ${ }^{2}$ P. K. Madhu, ${ }^{1}$ and Vipin Agarwal ${ }^{1, \text { a) }}$

1) TIFR Centre for Interdisciplinary Sciences, Tata Institute of

Fundamental Research, Sy. No. 36/P, Gopanpally, Hyderabad 500 107,

India

2) Physical Chemistry, ETH Zurich, Vladimir-Prelog-Weg 2, 8093 Zurich,

Switzerland

(Dated: 7 March 2019) 
Rotational-Echo DOuble Resonance, REDOR, is an experimentally robust and a well-established dipolar-recoupling technique to measure dipolar couplings between isolated pairs of spin-1/2 heteronuclei in solid-state Nuclear Magnetic Resonance (NMR). REDOR can also be used to estimate motional order parameters when the bond distance is known, for example, in the case of directly-bound nuclei. However, the relatively fast dipolar dephasing for strongly coupled spin-1/2 pairs, such as ${ }^{13} \mathrm{C}-{ }^{1} \mathrm{H}$, makes the stroboscopic measurement required in this experiment challenging, even at fast Magic-Angle-Spinning (MAS) frequencies. In such cases, modified REDOR-based methods like Shifted-REDOR (S-REDOR) are used to scale the dipolar coupling compared to REDOR. This is achieved by changing the position of one of the two recoupling $\pi$-pulses in a rotor period. This feature, however, comes at the cost of mixing multiple Fourier components of the dipolar coupling and can, additionally, require high Radio-Frequency ( $\mathrm{RF}$ ) amplitudes to realise small scaling factors. We introduce here a general pulse scheme which involves shifting both the $\pi$ pulses in the REDOR scheme to achieve arbitrary scaling factors whilst retaining the robustness and simplicity of REDOR recoupling and avoiding the disadvantages of S-REDOR. The classical REDOR is a specific case of this scheme with a scaling factor of one. We demonstrate the results on isolated ${ }^{13} \mathrm{C}-{ }^{15} \mathrm{~N}$ and ${ }^{1} \mathrm{H}-{ }^{13} \mathrm{C}$ spin pairs at 20 and $62.5 \mathrm{kHz}$ MAS, respectively.

Keywords: SSNMR, REDOR, S-REDOR, C-H dipolar couplings, Scaling factors

a)vipin@tifrh.res.in 
This manuscript was accepted by J. Chem. Phys. Click here to see the version of record.

\section{Publishi.hg INTRODUCTION}

Measuring heteronuclear dipolar coupling is an important way to determine the distance between two nuclei using solid-state Nuclear Magnetic Resonance (NMR). Rotational-Echo Double Resonance (REDOR) has been used to measure distances in a variety of systems ranging from materials to biomolecules and is also used to study reaction dynamics. ${ }^{1-8}$ Although experimentally a robust technique, mostly, only couplings in the range of 0.2 $2 \mathrm{kHz}$ have been reported at slow to moderate magic-angle-spinning (MAS) frequencies. ${ }^{3}$ This limitation is a direct result of the need to sample a sufficient number of points before and after the first dephasing minimum of the REDOR curve. Measurement of stronger couplings $(2-25 \mathrm{kHz})$ is challenging because the signal dephases very fast (for a typical onebond ${ }^{15} \mathrm{~N}-{ }^{1} \mathrm{H}$ coupling of $10.5 \mathrm{kHz}$, the signal dephases in less than $\left.200 \mu \mathrm{s}\right)$. The stroboscopic nature of sampling means that only four points can be obtained in the dephasing curve, even at $60 \mathrm{kHz}$ MAS frequency (the minimum sampling duration being $2 \tau_{\mathrm{r}}=33.33 \mu \mathrm{s}$ ). Thus, to measure such strong couplings accurately, modifications of the REDOR sequence that scale down the dipolar coupling are needed.

The focus of this manuscript is on the measurement of strong dipolar couplings. The REDOR sequence can be modified in several ways to scale down the effective dipolar coupling. ${ }^{1,9,10}$ The most common modification of the classical REDOR pulse scheme involves shifting the position of the $\pi$ pulse at half the rotor period by a fraction of the rotor period, $\tau_{\mathrm{r}}$, and is known as Shifted-REDOR (S-REDOR). This experiment was demonstrated in the original REDOR paper itself and has subsequently been refined by several groups. ${ }^{1,11,12}$ The resultant scaling factors in this experiment are a direct function of the shift of the first pulse and lower scaling factors typically require a larger shift in the pulse position. S-REDOR has two disadvantages: (i) In order to realise low scaling factors, higher Radio-Frequency (RF) amplitudes have to be used so that the two $\pi$ pulses do not overlap. (ii) S-REDOR leads to

a different shape of the dephasing curves due to the mixing of the $m=1$ and $m=2$ Fourier components of the dipolar Hamiltonian. ${ }^{10}$ The second method involves using homonuclear decoupling schemes on one of the spins to scale down the heteronuclear couplings. ${ }^{9}$ Here, the scaling factors are essentially fixed by the homonuclear decoupling sequence but may change substantially with experimental parameters, especially with the spinning frequency. A third method (SS-REDOR) involves using a scheme with the $\pi$ pulse positions based on a sine- 
Publishinggiared function. ${ }^{10}$ Low scaling factors in this scheme are achieved by increasing the cycle time of the sequence, which in turn restricts the minimum sampling duration. Thus, there is a need for a more general scheme based on REDOR that scales down dipolar couplings in a controllable manner without increasing the RF requirements and avoids mixing of the various Fourier components. Additionally, the scheme should retain the inbuilt robustness of REDOR to experimental mis-settings. Here, we present such a modified REDOR scheme.

We propose a new implementation of the S-REDOR sequence where both the $\pi$ pulses are shifted synchronously, i.e. the time duration between the two pulses always remains $0.5 \tau_{\mathrm{r}}$. The shift is implemented in a mirror-symmetric way during the dephasing period of the REDOR sequence. This scheme is shown to scale down the recoupled heteronuclear dipolar coupling Hamiltonian to any value without recoupling the second Fourier coefficient and has the same RF requirements as the REDOR experiment. The newly introduced scheme is robust in determining strong one-bond dipolar couplings such as ${ }^{15} \mathrm{~N}-{ }^{1} \mathrm{H}$ and ${ }^{13} \mathrm{C}-{ }^{1} \mathrm{H}$ at all MAS frequencies. We validate all the results on the well studied one-bond ${ }^{13} \mathrm{C}-{ }^{15} \mathrm{~N}$ dipolar coupling of $2-{ }^{13} \mathrm{C},{ }^{15} \mathrm{~N}$-Glycine at $20 \mathrm{kHz}$ MAS, and show its implementation for a strong one-bond ${ }^{13} \mathrm{C}-{ }^{1} \mathrm{H}$ coupling at a MAS frequency of $62.5 \mathrm{kHz}$, on a suitably labelled sample of Alanine. We also reyisit the interpretation of the original REDOR and S-REDOR experiment and show how the scaling of the dipolar coupling depends on the position of the pulses in the rotor period.

\section{THEORY}

In this section, we outline the schematic of the pulse sequences and evaluate them theoretically. Under MAS, the high-field heteronuclear dipole-dipole Hamiltonian becomes time dependent, as represented by

$$
\hat{\mathcal{H}}_{\mathrm{D}}(t)=2 \hat{I}_{z} \hat{S}_{z} \sum_{m=-2}^{2} \omega_{\mathrm{D}}^{(m)} e^{i m \omega_{\mathrm{r}} t}
$$

The MAS spinning frequency is denoted by $\omega_{\mathrm{r}}, m$ denotes the order of the component of

the second rank heteronuclear dipole-dipole coupling which varies from -2 to +2 , and $\omega_{\mathrm{D}}^{(m)}$ is defined as:

$$
\omega_{\mathrm{D}}^{(m)}=\frac{1}{2} d_{m, 0}^{2}\left(-\theta_{\mathrm{m}}\right) e^{-i m \gamma} d_{0, m}^{2}(\beta) \delta_{\mathrm{D}}
$$


Publishihgre $\beta$ and $\gamma$ are the Euler angles describing the orientation of crystallites in the powder sample in the rotor frame, $\theta_{\mathrm{m}}$ is the magic angle, $d_{m, m^{\prime}}^{2}(\beta)$ are the reduced Wigner rotation-matrix elements of rank 2 , and $\delta_{\mathrm{D}}$ is the anisotropy of the heteronuclear dipoledipole coupling between spins I and $\mathrm{S}$ with gyromagnetic ratios $\gamma_{I}$ and $\gamma_{S}$, respectively, and is defined as

$$
\delta_{\mathrm{D}}=-2 \frac{\mu_{0}}{4 \pi} \frac{\gamma_{I} \gamma_{S} \hbar}{r_{I S}^{3}}=2 b_{I S}
$$

To visualise the effect of $\pi$ pulses on the dipolar Hamilfonian, we plot the time evolution of the term corresponding to $m= \pm 1$ in Eq. $1\left(\mathcal{H}_{\mathrm{D}, \beta, \gamma}^{m= \pm 1}\right)$ under MAS (Fig. 1 ia) and three distinct placements of $\pi$ pulses (Fig. 1 ib-id) for one crystallite (Fig. 1 iia-d). As seen in Fig. 1 iia, MAS modulates the spatial part of the Hamiltonian with frequency $\pm 1 \omega_{\mathrm{r}}$. The average value of the dipolar Hamiltonian $\left(\overline{\mathcal{H}}_{\mathrm{D}, \beta, \tau}^{m= \pm 1}\right.$ and $\left.\overline{\mathcal{H}}_{\mathrm{D}, \beta, \tau}^{m= \pm 2}\right)$ over the duration of the two periods $(\mathrm{A}, \mathrm{B}$ and $\mathrm{A}+\mathrm{B})$ is zero, irrespective of the $\gamma$ angle (Fig. 1 iiia, iva). The $\pi$ pulses on one of the nuclei, in this case the non-observed nuclei, modulate the dipolar Hamiltonian via the spin part (Eq. 1). As shown in Fig. 1 iib-d, the Hamiltonian $\left(\mathcal{H}_{\mathrm{D}, \beta, \gamma}^{m= \pm 1}\right)$ is inverted after the $\pi$ pulses, assuming ideal delta pulses. This results in a non-zero average dipolar Hamiltonian over the A and B periods and forms the basis of dipolar recoupling in REDOR and related pulse sequences. A similar description for $m= \pm 2$ term in Eq. $1\left(\mathcal{H}_{\mathrm{D}, \beta, \gamma}^{m= \pm 2}\right)$ of the dipolar Hamiltonian is shown in Fig. S1.

In REDOR, the recoupled dipolar Hamiltonian depends critically on the placement of $\pi$ pulses. Several modifications of REDOR have been implemented to take advantage of the subtle differences in the samples under study. ${ }^{13-15}$ The mirror-symmetric implementation of REDOR (Fig. 1 ib) used in this study helps to visualise the various proposed modifications to the REDOR sequence. ${ }^{10}$ The symmetry referred here is with respect to the $\pi$ pulse on the I channel. All mirror-symmetric REDOR pulse sequences can be notated in a generalised way as $m \mathcal{R}_{\epsilon_{1}}^{\epsilon_{2}}$, where, $m$ refers to mirror-symmetry version, $\mathcal{R}$ to REDOR, $\epsilon_{1}$ and $\epsilon_{2}$ to the positions of the $\pi$ pulses as a fraction of $\tau_{\mathrm{r}}$. In this notation, the classical mirror-symmetric REDOR (as in Fig. 1 ib) may be notated as $m \mathcal{R}_{0.25}^{0.75}$.

The position of the $\pi$ pulses in a rotor period dictates the level of interference of spatial and spin modulations resulting in scaled recoupling. For example, consider $m \mathcal{R}_{0.10}^{1.0}$ as in Fig. 1 ic which belongs to the mirror-symmetric S-REDOR family. The $m \mathcal{R}_{0.10}^{1.0}$ sequence modulates the amplitude and phase of the average dipolar coupling Hamiltonian in periods 
Publishifgand B, as shown in Fig. 1 iiic. However, the phase modulation is averaged over the combined $\mathrm{A}$ and $\mathrm{B}$ time periods leading to only the amplitude-modulated recoupled nonzero, $\overline{\mathcal{H}}_{\mathrm{D}, \beta, \tau}^{m= \pm 1}$ (Fig. 1 iiic) and $\overline{\mathcal{H}}_{\mathrm{D}, \beta, \tau}^{m= \pm 2}$ (Fig. 1 ivc) terms. We define the scaling factor $\kappa$ as the ratio of the recoupled $\overline{\mathcal{H}}_{\mathrm{D}, \beta, \tau_{\mathrm{A}+\mathrm{B}}}^{m= \pm 1}$ terms achieved by any modified REDOR sequence to that of the classical REDOR sequence. The $m \mathcal{R}_{\epsilon_{1}}^{\epsilon_{2}}$ sequence will also recouple the $\overline{\mathcal{H}}_{\mathrm{D}, \beta, \tau}^{m= \pm 2}$ except when $\left|\epsilon_{1}-\epsilon_{2}\right|=0.5$ and for $m \mathcal{R}_{0.25}^{1.0}, m \mathcal{R}_{0.75}^{1.0}$ and $m \mathcal{R}_{0.25}^{0.50}$, where mirror symmetry ensures no recoupling of $\overline{\mathcal{H}}_{\mathrm{D}, \beta, \tau}^{m= \pm 2}$. The two Fourier components have a very different crystal dependence and contribute differently to scaling factors. As shown further below, in Fig. 4a$\mathrm{d}$, the position of the first dipolar oscillation is primarily determined by $\overline{\mathcal{H}}_{\mathrm{D}, \beta, \tau}^{m= \pm 1}$ while $\overline{\mathcal{H}}_{\mathrm{D}, \beta, \tau}^{m= \pm 2}$ changes the intensity and dampen the dipolar oscillation of the REDOR build-up curves. ${ }^{10}$ Therefore, we use $\overline{\mathcal{H}}_{\mathrm{D}, \beta, \tau_{\mathrm{A}+\mathrm{B}}}^{m= \pm 1}$ to define $\kappa$.

The pulse scheme of the new REDOR sequence is designated as $m \mathcal{R}_{\epsilon}^{0.5+\epsilon}$ and is shown in (Fig. $1 \mathrm{id}$ ). Here, the $\pi$ pulses are always separated by $0.5 \tau_{\mathrm{r}}$ in a rotor period and are simultaneously moved to obtain scaling of the recoupled dipolar coupling Hamiltonian. Due to the pulse spacing by $0.5 \tau_{\mathrm{r}}$, only $\overline{\mathcal{H}}_{\mathrm{D}, \beta, \tau}^{m= \pm 1}$ is recoupled, as can be seen in Fig. 1 iiid, ivd. Moving the pulses towards the beginning of the rotor period in block $\mathrm{A}$ and towards the end in block $\mathrm{B}$ whilst maintaining the constant separation of $0.5 \tau$ between any two pulses of a block only modulates the phase (Fig. 1 iiid) of the $\overline{\mathcal{H}}_{\mathrm{D}, \beta, \tau}^{m= \pm 1}$ term. It is the combined average of $\overline{\mathcal{H}}_{\mathrm{D}, \beta, \tau}^{m= \pm 1}$ over the $\mathrm{A}$ and $\mathrm{B}$ recoupling periods that results in scaled recoupled Hamiltonian (Fig. 1 iiid). In this scheme, irrespective of the position of the first $\pi$ pulse, $\overline{\mathcal{H}}_{\mathrm{D}, \beta, \tau}^{m= \pm 2}$ (Fig. 1 ivd) is never recoupled.

$m \mathcal{R}_{0.25}^{0.75}$ and $m \mathcal{R}_{\epsilon}^{0.5+\epsilon}$ schemes always have a minimum spacing of $0.5 \tau_{\mathrm{r}}$ between the center of the two $\pi$ pulses and, therefore, a minimum RF amplitude of $1.0 \omega_{\mathrm{r}}$ is required. In contrast, in $m \mathcal{R}_{\epsilon_{1}}^{1.0}$ sequence $\pi$ pulses are applied at $\epsilon_{1} \tau_{\mathrm{r}}$ and $1.0 \tau_{\mathrm{r}}$. Moving the $\pi$ pulse closer to the beginning of a rotor period whilst applying another $\pi$ pulse at the end of the previous rotor period increases the RF requirement. The increased RF requirement depends on how $\epsilon_{1}$ is changed. For the $m \mathcal{R}_{\epsilon_{1}}^{1.0}$ sequence, $\kappa$ in the range of 0 to 0.5 can be achieved by choosing $\epsilon_{1}$ between $0-0.25$ or $0.25-0.5$. However, for $\epsilon_{1}$ ranging from $0-0.25$ the RF amplitude becomes prohibitively high, whilst for $\epsilon_{1}$ ranging from $0.25-0.5$ the maximum $\mathrm{RF}$ requirement is only doubled. The $m \mathcal{R}_{\epsilon_{1}}^{1.0}$ sequence can also be implemented as $m \mathcal{R}_{\epsilon_{1}}^{0.5}$ sequence and are shown below to be equivalent. Fig. 2 shows $\overline{\mathcal{H}}_{\mathrm{D}, \beta, \tau}^{m= \pm 1}$ and $\overline{\mathcal{H}}_{\mathrm{D}, \beta, \tau}^{m= \pm 2}$ terms as a function of $\gamma$ for different values of $\epsilon_{1}$. We can see that when $\epsilon_{1}$ is 0.25 , both these schemes do not recouple $\overline{\mathcal{H}}_{\mathrm{D}, \beta, \tau}^{m= \pm 2}$ 
Publishimgrn and $\overline{\mathcal{H}}_{\mathrm{D}, \beta, \tau}^{m= \pm 1}$ term is scaled by 0.5 (Fig. 2a,d). Secondly, shifting the $0.25 \tau_{r}$ pulse either way leads to further reduction in the recoupling of $\overline{\mathcal{H}}_{\mathrm{D}, \beta, \tau}^{m= \pm 1}$ and increase in $\overline{\mathcal{H}}_{\mathrm{D}, \beta, \tau}^{m= \pm 2}$, as evident from Fig. 2b, c, e, f. All these conditions are equivalent in terms of recoupling the Fourier components and scaling factor. The scaling is the same at all $0.25 \pm \delta$ positions, implying no differentiation between moving the $\pi$ pulse towards or away from the beginning of the rotor period. Thus, we can conclude that, the schemes $m \mathcal{R}_{\epsilon_{1}}^{0.5}$ and $m \mathcal{R}_{\epsilon_{1}}^{1.0}$ are equivalent and it is advantageous to use $m \mathcal{R}_{\epsilon}^{0.5}$ for scaling if we consider moving $\pi$ pulse towards the beginning of rotor period.

The pulse sequence incorporating $m \mathcal{R}_{\epsilon}^{0.5+\epsilon}$ for ${ }^{13} \mathrm{C}-{ }^{1} \mathrm{H}$ dipolar recoupling experiments with cross-polarisation from ${ }^{1} \mathrm{H}$ to ${ }^{13} \mathrm{C}$ is shown in Fig. $3 .{ }^{16}$ The scheme employs $\tau_{0}$ and $\tau_{\mathrm{r}}$ - $\tau_{0}$ periods, one in each block, for positioning of $\pi$ pulses without enforcing RF restrictions. This implementation allows using $2 n \tau_{\mathrm{r}}$ duration for application of frequency-selective pulses at the center of the sequence. Only the $\tau_{0}$ period is changed to achieve the desired scaling.

We now consider the REDOR schemes more quantitatively using average Hamiltonian theory (AHT).${ }^{17-20}$ The first-order average Hamiltonian $\left(\overline{\mathcal{H}}_{\mathrm{D}}^{(1)}\right)$ for $m \mathcal{R}_{0.25}^{0.75}$ considering real pulses under the XY-4 phase-cycling scheme-is

$$
\overline{\mathcal{H}}_{\mathrm{D}}^{(1)}=\left(\frac{\cos \left(\frac{\pi \phi}{2}\right)}{1-\phi^{2}}\right) \frac{\sqrt{2}}{\pi} b_{I S} \cos (\gamma) \sin (2 \beta) 2 \hat{I}_{z} \hat{S}_{z}
$$

where, $\phi=\frac{2 \tau_{\mathrm{p}}}{\tau_{\mathrm{r}}}$, with $/ \tau_{\mathrm{p}}$ the duration of the $\pi$ pulse. $^{21}$ The term $\frac{\cos \left(\frac{\pi \phi}{2}\right)}{1-\phi^{2}}$ is the scaling, $\kappa_{\phi}$, caused by the finite pulse width, $\phi .{ }^{22}$ The average Hamiltonian contains contribution only from the first Fourier component.

The first-order average Hamiltonian for $m \mathcal{R}_{\epsilon_{1}}^{1.0}$ with finite-width pulses under XY-4 scheme is

$$
\begin{aligned}
\overline{\mathcal{H}}_{\mathrm{D}}^{(1)}= & \frac{\sin (2 \pi \epsilon)}{2} \kappa_{\phi} \frac{\sqrt{2}}{\pi} b_{I S} \cos (\gamma) \sin (2 \beta) 2 \hat{I}_{z} \hat{S}_{z} \\
& +\frac{\cos (\pi \phi)}{1-4 \phi^{2}} \frac{\sin (4 \pi \epsilon)}{2} \frac{1}{2 \pi} b_{I S} \cos (2 \gamma) \sin ^{2}(\beta) 2 \hat{I}_{z} \hat{S}_{z}
\end{aligned}
$$

This average Hamiltonian contains contribution from $\omega_{\mathrm{D}}^{( \pm 1)}$ (first term) and $\omega_{\mathrm{D}}^{( \pm 2)}$ (second term), where $\epsilon=\epsilon_{1} \cdot{ }^{12}$ The coefficient of the $\omega_{\mathrm{D}}^{( \pm 1)}$ term has scaling contributions from finite width of pulses, $\kappa_{\phi}$, and position of the $\pi$ pulse, $\kappa=\frac{\sin (2 \pi \epsilon)}{2} . \kappa$ is the desired scaling term multiplying the first Fourier component and depends only on the pulse position $\epsilon$. The average Hamiltonian analysis for $m \mathcal{R}_{\epsilon_{1}}^{0.5}$ is the same as for $m \mathcal{R}_{\epsilon_{1}}^{1.0}$ given in Eq. 5. This is not 
Publishing rising as the purpose of the second $\pi$ pulse in a rotor period is to reorient the dipolar Hamiltonian for recoupling over the next rotor period.

The first-order average Hamiltonian for the scheme $m \mathcal{R}_{\epsilon}^{0.5+\epsilon}$ considering real pulses under $\mathrm{XY}-4$ scheme is given as:

$$
\overline{\mathcal{H}}_{\mathrm{D}}^{(1)}=\sin (2 \pi \epsilon) \kappa_{\phi} \frac{\sqrt{2}}{\pi} b_{I S} \cos (\gamma) \sin (2 \beta) 2 \hat{I}_{z} \hat{S}_{z}
$$

We know from the Fourier picture that positioning the $\pi$ pulses $0.5 \tau_{\mathrm{r}}$ apart does not recouple the $\omega_{\mathrm{D}}^{( \pm 2)}$ term which is also confirmed by the AHT calculations shown in Eq. 6. This average Hamiltonian has the same functional form as the one of the original REDOR experiment with an additional scaling term $\sin (2 \pi \epsilon)$.

Fig. 4a compares the $m \mathcal{R}_{\epsilon_{1}}^{1.0}$ build-up curves showing the influence of $\omega_{\mathrm{D}}^{( \pm 1)}$ and $\omega_{\mathrm{D}}^{( \pm 2)}$ by delienating them from Eq. 5. For $\epsilon_{1}=0.25$, the $\omega_{D}^{( \pm 2)}$ term is absent and REDOR like dipolar oscillations are visible. However, in Fig. 4b-d, $\omega_{\mathrm{D}}^{( \pm 1)}$ and $\omega_{\mathrm{D}}^{( \pm 2)}$ interfere with each other and dampen the oscillations. The amount of dampening depends on the amplitude of the recoupled Fourier components which is controlled by the position of the $\pi$ pulses in a rotor period. ${ }^{10}$ The lower the scaling achieved by $m \mathcal{R}_{\epsilon_{1}}^{1.0}$ or $m \mathcal{R}_{\epsilon_{1}}^{0.5}$, the larger will be the deviation from an ideal REDOR curve. Further, the mixing also leads to an overshoot in intensity beyond 1.04 unlike in the original REDOR curve or $m \mathcal{R}_{\epsilon}^{0.5+\epsilon}$. Fig. 4e-g show the dependence of the recoupled Hamiltonian for the different schemes as a function of $\gamma$ and $\beta$ angles (crystal orientation) for different sequences. Fig. 4e,g are for the schemes $m \mathcal{R}_{0.25}^{0.75}$ and $m \mathcal{R}_{0.05}^{0.55}$, respectively. The $\beta$ dependence of $m \mathcal{R}_{0.25}^{0.75}$ (i.e. $\left.\sin (2 \beta)\right)$ is maintained in $m \mathcal{R}_{0.05}^{0.55}$ whilst $m \mathcal{R}_{0.1}^{0.5}$ (Fig. 4f) has the superposition of $\sin (2 \beta)$ and $\sin ^{2}(\beta)$ terms.

The scaling term $\kappa$ in the average Hamiltonian for $m \mathcal{R}_{\epsilon_{1}}^{1.0}$ (or $m \mathcal{R}_{\epsilon_{1}}^{0.5}$ ) and $m \mathcal{R}_{\epsilon}^{0.5+\epsilon}$, is $\frac{\sin (2 \pi \epsilon)}{2}$ and $\sin (2 \pi \epsilon)$, respectively. The $\omega_{\mathrm{D}}^{( \pm 2)}$ term in $m \mathcal{R}_{\epsilon_{1}}^{1.0}$ schemes are scaled by $\frac{\sin (4 \pi \epsilon)}{2}$ and predominantly contribute in sequences with lower scaling factors. These functions are periodic and monotonically increase (Fig. 5a) between $\epsilon$ values 0 to 0.25 , thereby defining the bounds on the pulse position. From Fig. 5a it is evident that $m \mathcal{R}_{\epsilon_{1}}^{1.0}$ and $m \mathcal{R}_{\epsilon}^{0.5+\epsilon}$ have a scaling factor range of 0 to 0.5 and 0 to 1.0, respectively. The form of $\kappa_{\phi}$ remains the same in all the average Hamiltonains. $\kappa_{\phi}=\frac{\cos (\pi \phi / 2)}{1-\phi^{2}}$ can scale down the dipolar coupling by approximately $22 \%$ (Fig. $5 \mathrm{~b}$ ) when $\pi$ pulses fill the rotor period. ${ }^{23}$ The prefactor $\frac{\cos (\pi \phi)}{1-4 \phi^{2}}$ of the recoupled $\omega_{\mathrm{D}}^{( \pm 2)}$ term in $m \mathcal{R}_{\epsilon_{1}}^{1.0}$ scheme will always have a non-zero (Fig. $5 \mathrm{c}$ ) value irrespective of the value of $\phi$ and is more dramatically scaled compared to the $\omega_{\mathrm{D}}^{( \pm 1)}$ term. 
Publishing The pulse schemes were analysed using numerical SIMPSON simulations. ${ }^{24,25}$ Details of the simulations are presented in the numerical simulation section. Fig. 5d shows the dependence of scaling factor $\kappa$ on $\epsilon$ for schemes $m \mathcal{R}_{\epsilon}^{0.5}$ and $m \mathcal{R}_{\epsilon}^{0.5+\epsilon}$. These two curves replicate the result obtained from the average Hamiltonian calculations, as shown in Fig. 5a. In addition, we evaluated the scaling dependence on the pulse width $\phi$ (Fig. 5e) and the results match exactly with the theoretical calculations (Fig. 5b). Fig. 5f shows the build-up profiles of $m \mathcal{R}_{0.25}^{0.75}$ for a coupling of $1000 \mathrm{~Hz}$ with $\phi$ values of $0.01,0.5$ and 1.0. The figure clearly shows the scaling of the build-up profiles because of pulse widths effect, $\phi$. Fig. $5 \mathrm{~g}$ shows build-up profiles of $m \mathcal{R}_{\epsilon_{1}}^{1.0}$ (line) and $m \mathcal{R}_{\epsilon_{1}}^{0.5}$ (dotted) for $\epsilon_{1}$ values of $0.25,0.20$ and 0.10. Fig. $5 \mathrm{~g}$ shows the effects of mixing of the $\omega_{\mathrm{D}}^{( \pm 1)}$ and $\omega_{\mathrm{D}}^{( \pm 2)}$ components on the REDOR build-up profiles. Moving the pulse from $0.25 \tau_{\mathrm{r}}$ to $0.20 \tau_{\mathrm{r}}$ in $m \mathcal{R}_{\epsilon_{1}}^{0.5}$ results in a very small change in the scaling factor but introduces the $\omega_{\mathrm{D}}^{( \pm 2)}$ component (as seen from red and green curves). Moving the pulse further to $0.10 \tau_{\mathrm{r}}$ has a dramatic effect. The magnitude of the curve exceeds 1.04 and loses the oscillations in the profile. These effects do not seem to have much impact on fitting the experimental data using SIMPSON simulations but lack the aesthetic visual elucidation of the frequency from oscillations. Fig. 5h shows build-up profiles for various $\kappa(1.0,0.71,0.51,0.31,0.125)$ values obtained with $m \mathcal{R}_{\epsilon}^{0.5+\epsilon}$ scheme by varying the $\epsilon(0.25,0.125,0.085,0.05,0.02)$ values. The scaling factor $\kappa$ varies from 1 to 0 as predicted from theory and the oscillations are maintained irrespective of the pulse position $\epsilon$. Fig. 5i shows the RF amplitude required to obtain a particular scaling factor from $m \mathcal{R}_{\epsilon_{1}}^{0.5}$ and $m \mathcal{R}_{\epsilon}^{0.5+\epsilon}$. Since, the two pulses in $m \mathcal{R}_{\epsilon}^{0.5+\epsilon}$ are always positioned $0.5 \tau_{\mathrm{r}}$ apart there is no $\mathrm{RF}$ dependence on $\epsilon$. Further, RF requirement is the same as that of REDOR (minimum $\mathrm{RF}$ required is $1.0 \omega_{\mathrm{r}}$ ) for all $\epsilon$ values. In the context of this study, it is important to note that the proposed $m \mathcal{R}_{\epsilon}^{0.5+\epsilon}$ can sequence can be used to scale down the dipolar Hamiltonian to any value without worrying about the RF amplitude.

$m \mathcal{R}_{\epsilon}^{0.5+\epsilon}$ can also be implemented by constituting a basic recoupling block over two rotor periods where A and B blocks are alternated. In first-order AHT, the performance of this scheme will remain the same but there will be changes in higher-order terms since the compensation of the two blocks happens on a much shorter time scale. Such an implementation would increase the minimum sampling time from $2 \tau_{\mathrm{r}}$ to $4 \tau_{\mathrm{r}}$. In addition, it would increase the minimum RF amplitude requirements since the pulses from B and A block move toward the same time point for low $\epsilon$ values. 
Publishing summarise, the $m \mathcal{R}_{\epsilon}^{0.5+\epsilon}$ scheme recouples only the $\omega_{\mathrm{D}}^{( \pm 1)}$ component and allows a scaling of dipolar couplings over the full range from 1 to 0 . In contrast, $m \mathcal{R}_{\epsilon_{1}}^{\epsilon_{2}}$ schemes recouple both the $\omega_{\mathrm{D}}^{( \pm 1)}$ and $\omega_{\mathrm{D}}^{( \pm 2)}$ components except when $\left|\epsilon_{1}-\epsilon_{2}\right|=0.5$ or specifically for $m \mathcal{R}_{0.25}^{0.5}, m \mathcal{R}_{0.25}^{1.0}, m \mathcal{R}_{0.75}^{1.0}$ schemes and the scaling factor range is restricted between 0.5 to 0 . However, the S-REDOR sequences can also be implemented in an asymmetric manner to achieve scaling factors from 1 to 0 . Also the RF requirements for the $m \mathcal{R}_{\epsilon}^{0.5+\epsilon}$ is the same as for the original REDOR sequence while $m \mathcal{R}_{\epsilon_{1}}^{\epsilon_{2}}$ could require up to two times the RF amplitudes of the $m \mathcal{R}_{\epsilon}^{0.5+\epsilon}$ sequence. Further, $m \mathcal{R}_{\epsilon}^{0.5+\epsilon}$ scheme has similar tolerance to misset in $\pi$ pulse width as REDOR (Fig. S2). These properties of $m \mathcal{R}_{\epsilon}^{0.5+\epsilon}$ make it ideal for applications at all MAS frequencies.

\section{RESULTS AND DISCUSSION}

The above arguments were tested on an ideal two-spin system of $2-{ }^{13} \mathrm{C},{ }^{15} \mathrm{~N}$-Glycine at a moderate MAS frequency of $20 \mathrm{kHz} . m \mathcal{R}_{\epsilon_{1}}^{0.5}$ and $m \mathcal{R}_{\epsilon}^{0.5+\epsilon}$ experiments were performed for $\epsilon$ values of $0.25,0.20,0.10$ and $0.25,0.125,0.085$ and 0.05 respectively. Experimental $\Delta S / S_{0}$ profiles and SIMPSON fits are plotted for $m \mathcal{R}_{\epsilon_{1}}^{0.5}$ (Fig. 6a) and $m \mathcal{R}_{\epsilon}^{0.5+\epsilon}$ (Fig. 6b). The fit parameters for all these schemes are given in Table I. Experimental results agree with simulation and theory. Further, the ability of the pulse scheme to incorporate additional pulse elements is demonstrated here with frequency selective pulses, Fig. 7, on either or both $\mathrm{C}$ and $\mathrm{N}$ channels for representative $\epsilon$ values. The figure compares the build-up curves for selective and hard pulses. The rate of the build-up is the same whereas there is a slight increase in the intensity of the build-up with the selective pulses. We believe that this effect is because of/measuring the data points further out with the $T_{2}^{\prime}$ profile. The purpose of employing selective pulses is to show that inclusion of very long pulses, spanning several rotor periods, does not alter the REDOR profile, discounting $T_{2}^{\prime}$ effects .

For strong coupling measurement, we chose deuterated alanine having only $\mathrm{H}_{\alpha}-\mathrm{C}_{\alpha}$ labelled with ${ }^{13} \mathrm{C}$ (see Fig. S3 for labelling scheme) having a heteronuclear dipolar coupling of ca. $21.5 \mathrm{kHz}$. Deuteration reduces the ${ }^{1} \mathrm{H}-{ }^{1} \mathrm{H}$ homonuclear dipolar couplings. $m \mathcal{R}_{0.25}^{0.75}$ (Fig. 8a), $m \mathcal{R}_{\epsilon}^{0.5}$ (Fig. $8 \mathrm{~b}, \mathrm{c}, \mathrm{d}$ ) and $m \mathcal{R}_{\epsilon}^{0.5+\epsilon}$ (Fig. $8 \mathrm{e}, \mathrm{f}, \mathrm{g}, \mathrm{h}$ ) were performed at a MAS frequency of $62.5 \mathrm{kHz}$ (with $\tau_{\mathrm{r}}=16 \mu \mathrm{s}$ ) and corresponding $\Delta S / S_{0}$ curves with fits are plotted in Fig. 8 . We can clearly see the justification for the requirement of scaling from the REDOR data 
Publishinfgig. 8a). There are only three points in the $\Delta S / S_{0}$ build up of $m \mathcal{R}_{0.25}^{0.75}$ and are not sufficient for proper mapping of the build-up profile. $m \mathcal{R}_{\epsilon_{1}}^{0.5}$ (Fig. 8b, c, d) with $\epsilon$ values $0.25,0.20$ and 0.10 (corresponding $\kappa$ is $0.50,0.49$ and 0.34 ) scales the build-up but the curves get distorted as expected with as $\epsilon$ changes from 0.25. But, the features of the experimental curves can be reproduced in the fits. Experimental $\Delta S / S_{0}$ curves are plotted for $m \mathcal{R}_{\epsilon}^{0.5+\epsilon}$ (Fig. 8e, f, g, h) with $\epsilon$ values $0.125,0.085,0.05$ and 0.02 (with $\kappa$ values $0.71,0.51,0.31$ and 0.125). The curves maintain at least three oscillations and the oscillation frequency from experiments matches exactly with the fits. We can also see that the estimated coupling in case of $m \mathcal{R}_{\epsilon}^{0.5+\epsilon}$ with $\kappa$ values more than 0.3 agree well whereas there is a large deviation in case of $m \mathcal{R}_{\epsilon}^{0.5}$ with similar $\kappa$ values. $m \mathcal{R}_{\epsilon}^{0.5+\epsilon}$, hence, can scale down to atleast 0.3 without error in the fits in case of $\mathrm{C}-\mathrm{H}$ couplings.

\section{A. Numerical simulations}

All the simulations were performed using the SIMPSON NMR simulation package. ${ }^{24,25}$ For evalutating the scaling factors (as in Fig. 5) of the schemes a two spin ${ }^{13} \mathrm{C}-{ }^{15} \mathrm{~N}$ system with a dipolar coupling of $1000 \mathrm{~Hz}$ was considered. The simulations were performed at a MAS frequency of $20 \mathrm{kHz}$ considering ideal, infinitely strong $\pi$ pulses with 320 crystallites defined by the REPULSION/scheme and $16 \gamma$-angles. ${ }^{26}$ A series of $\Delta S / S_{0}$ profiles of REDOR was simulated by varying the dipolar coupling from 1 to $1000 \mathrm{~Hz}$ in steps of 1 . Another series of $\Delta S / S_{0}$ profiles with dipolar coupling of $1000 \mathrm{~Hz}$ for all the modified REDOR schemes were simulated varying $\epsilon_{1}$ and $\epsilon_{2}$. Then, the scaled dipolar coupling is the best fit of each $\Delta S / S_{0}$ profiles with that of REDOR $\Delta S / S_{0}$ profiles. Scaling factor $\kappa$ from the simulations is the ratio of scaled dipolar coupling from the best fit to actual dipolar coupling of the simulation $(1000 \mathrm{~Hz})$. Only the first $30 \%$ of the $\Delta S / S_{0}$ build-up was considered for the best fits (because of deviations in $m \mathcal{R}_{\epsilon_{1}}^{0.5}$ ). For evaluating the scaling factors associated with the finiteness of the $\pi$ pulse, a similar exercise was carried out. The $\Delta S / S_{0}$ profiles corresponding to each of the REDOR schemes were simulated with delta pulses by varying the dipolar coupling from 1 to $1000 \mathrm{~Hz}$. Another set of simulations carried out by varying $\phi$ was then fitted to $\Delta S / S_{0}$ profiles with infinitely strong pulses to obtain the scaling factors. 
This manuscript was accepted by J. Chem. Phys. Click here to see the version of record.

\section{Experimental details}

Experiments on an ideal two-spin system of $2-{ }^{13} \mathrm{C},{ }^{15} \mathrm{~N}$-Glycine sample (obtained from Cambridge Isotopes) were carried out in a $2.5 \mathrm{~mm}$ triple-resonance Bruker MAS probe on a $700 \mathrm{MHz}$ Bruker Avance-III spectrometer. All the experiments were performed at $20 \mathrm{kHz}$ spinning frequency with ${ }^{13} \mathrm{C}$ and ${ }^{15} \mathrm{~N} \pi$ pulse durations of $10 \mu \mathrm{s}$ and $7.6 \mu \mathrm{s}$, respectively. Experiments were implemented in a pseudo two-dimensional fashion with a recycle delay of $3.0 \mathrm{~s}$ and acquiring 32 scans for signal averaging. Uninterrupted XY-8 phase cycling was applied on the I or S channel over the complete evolution period. Continuous-Wave (CW) decoupling with $90 \mathrm{kHz}$ RF amplitude was applied on ${ }^{1} \mathrm{H}$ during the REDOR evolution and $\mathrm{rCW}^{A p A}$ decoupling of $90 \mathrm{kHz} \mathrm{RF}$ amplitude was applied on ${ }^{1} \mathrm{H}$ during the acquisition. ${ }^{27}$ For the reference $\left(S_{0}\right)$ profile spin-echo experiments were performed over the complete recoupling duration. For the experiments with selective inversion pulses the $\pi$ pulse lengths were optimised to $1.0 \mathrm{~ms}$ width which resulted in a 22 rotor-period central block $\left(2 n \tau_{\mathrm{r}}\right.$ in Fig. 3$)$.

${ }^{13} \mathrm{C}-{ }^{1} \mathrm{H}$ REDOR experiments were performed on an L-Alanine $\left(3,3,3, \mathrm{~N}, \mathrm{~N}, \mathrm{~N}-\mathrm{D}_{6}-2-{ }^{13} \mathrm{C},{ }^{15} \mathrm{~N}\right)$ (referred as deuterated alanine, shown in Fig. S3) sample containing only ${ }^{1} \mathrm{H}_{\alpha^{-}}{ }^{13} \mathrm{C}_{\alpha}$ spin pairs in a $1.3 \mathrm{~mm}$ MAS probe on a $700 \mathrm{MHz}$ Bruker Avance-III spectrometer. All experiments were performed at $\omega_{\mathrm{r}}$ of $62.5 \mathrm{kHz}$ with $2.0 \mu \mathrm{s}$ and $3.84 \mu \mathrm{s} \pi$ pulses on ${ }^{1} \mathrm{H}$ and ${ }^{13} \mathrm{C}$ respectively. For heteronuclear decoupling, low-power $\mathrm{rCW}^{A p A}$ with $12 \mathrm{kHz} \mathrm{RF}$ amplitude was applied on ${ }^{1} \mathrm{H}$ during the acquisition. ${ }^{28}$

All experimental data were processed with NMRPipe suite and intensities were obtained by integrating the area under respective peaks. ${ }^{29}$ The dipolar couplings were estimated by fitting the experimental data to SIMPSON data by varying dipolar coupling and an intensity multiplier c. SIMPSON $\Delta S / S_{0}$ curves for each scheme with dipolar coupling range of 600 to $1000 \mathrm{~Hz}$, increment of $1 \mathrm{~Hz}$ for C-N and $16000 \mathrm{~Hz}$ to $25000 \mathrm{~Hz}$ with a increment of $20 \mathrm{~Hz}$ for $\mathrm{H}_{-} \mathrm{C}$ were multiplied with $\mathrm{c}$ and the whole 2-D grid was used for the fitting of each of the experimental profiles. 320 crystallites defined by REPULSION scheme and $64 \gamma$-angles were chosen for these simulations. The experimental data up to the first oscillation were chosen from each of the curves for fitting. The value of the parameter c from best fit is 1.06, 1.07, 1.05, 1.00, 1.07, 1.07, 1.07 and 1.06 for $m \mathcal{R}_{0.25}^{0.75}$ (REDOR), $m \mathcal{R}_{0.25}^{0.5}, m \mathcal{R}_{0.2}^{0.5}, m \mathcal{R}_{0.1}^{0.5}, m \mathcal{R}_{0.125}^{0.625}$, $m \mathcal{R}_{0.085}^{0.585}, m \mathcal{R}_{0.05}^{0.55}$ and $m \mathcal{R}_{0.02}^{0.52}$ respectively. This parameter corrects for the intensity errors arising from a combination of pulse imperfections, RF field inhomogeneity and rotor angle 
Publishimgors. All the figures were made using Gnuplot 5.0 and edited using inkscape 0.92.

\section{CONCLUSIONS}

We have proposed here a two-pulse shifted REDOR scheme encompassing the classical REDOR as a specific condition. The generalised REDOR scheme, $m \mathcal{R}_{\epsilon}^{0.5+\epsilon}$, is experimentally robust as the classical REDOR scheme and additionally allows arbitrary scaling down of the recoupled heteronuclear dipolar Hamiltonian. Further, this scheme is flexible enough to incorporate frequency-selective long pulses for various applications. We are working towards understanding the effect of ${ }^{1} \mathrm{H}-{ }^{1} \mathrm{H}$ homonuclear couplings whilst measuring the heteronuclear couplings and ways to minimise this effect.

We additionally show an alternate way to implement the already known symmetric SREDOR. A particular solution of this scheme $m \mathcal{R}_{0.25}^{1.0}$ or $m \mathcal{R}_{0.25}^{0.5}$ has scaling factor of 0.5 and has all the desirable characteristics of REDOR. A disadvantage of $m \mathcal{R}_{0.25}^{0.5}$ is that the $\mathrm{RF}$ amplitude requirement is twice the MAS frequency.

\section{ACKNOWLEDGEMENT}

We thank Manoj Naik and D. Krishna Rao for technical assistance. KRM acknowledges Department of Science and Technology (DST), India, for support under the Inspire Faculty Programme and TCIS, TIFR Hyderabad, for support under the Young Researcher Programme. VA acknowledges support from DST via grant number ECR/2017/001450. The National Facility for High Field NMR, TIFR Hyderabad, is acknowledged for NMR spectrometer access. This work was supported by the Swiss National Science Foundation (Grant No. 200020 169879) and the ETH Zurich (Grant No. ETH-10 15-1).

\section{FIGURES AND TABLES}


1. Pulse schematics for ia) MAS alone, ib) $m \mathcal{R}_{0.25}^{0.75}$, ic) $m \mathcal{R}_{0.1}^{1.0}$ and id) $m \mathcal{R}_{0.05}^{0.55}$. iia - iid) Time evolution of the first Fourier component of the dipolar coupling Hamiltonian $\left(\mathcal{H}_{\mathrm{D}, \beta=\frac{\pi}{4}, \gamma=0}^{m= \pm 1}\right)$ in the interaction frame under the effect of MAS and recoupling $\pi$ pulses for schemes listed in panel i). iiia-iiid) Integrals of the first Fourier term $\left(\overline{\mathcal{H}}_{\mathrm{D}, \beta, \tau}^{m= \pm 1}\right)$ and iva-ivd) the second Fourier term $\left(\overline{\mathcal{H}}_{\mathrm{D}, \beta, \tau}^{m= \pm 2}\right)$ of the dipolar coupling Hamiltonian in panel ii) as a function of the $\gamma$ angle, individually and cumulatively over the rotor periods A and B for all schemes in panel i).

FIG. 2. Individual and cumulative integral of the first $\left(\overline{\mathcal{H}}_{\mathrm{D}, \beta, \tau}^{m= \pm 1}\right)$ and second $\left(\overline{\mathcal{H}}_{\mathrm{D}, \beta, \tau}^{m= \pm 2}\right)$ Fourier terms of the heteronuclear dipolar coupling terms for a) $m \mathcal{R}_{0.25}^{1.0}$, b) $m \mathcal{R}_{0.1}^{1.0}$, c) $m \mathcal{R}_{0.4}^{1.0}$, d) $m \mathcal{R}_{0.25}^{0.5}$, e) $m \mathcal{R}_{0.1}^{0.5}$ and f) $m \mathcal{R}_{0.4}^{0.5}$ sequences as a function of the $\gamma$ angle over different recoupling periods. The blue curves denote the corresponding REDOR profiles for the two terms as reference.

FIG. 3. ${ }^{13} \mathrm{C}-{ }^{1} \mathrm{H}$ REDOR pulse sequence incorporating $m \mathcal{R}_{\epsilon}^{0.5+\epsilon}$.

FIG. 4. Effect of recoupling $\omega_{\mathrm{D}}^{( \pm n)}$ in $m \mathcal{R}_{\epsilon_{1}}^{0.5}$ scheme with $\epsilon_{1}$ values of a) 0.25 , b) 0.20 , c) 0.15 and d) 0.10. The red curve contains contributions from both $\omega_{\mathrm{D}}^{( \pm 1)}$ and $\omega_{\mathrm{D}}^{( \pm 2)}$ and the blue curves contain contribution only from the $\omega_{\mathrm{D}}^{( \pm 1)}$ component of the recoupled dipolar coupling Hamiltonian. Crystal orientation dependence of the recoupled dipolar Hamiltonian for e) $m \mathcal{R}_{0.25}^{0.75}$ (REDOR), f) $m \mathcal{R}_{0.10}^{0.5}$ $\left(m \mathcal{R}_{0.10}^{1.0}\right)$ and $\left.\mathrm{g}\right) m \mathcal{R}_{0.05}^{0.55}$ schemes. The differential intensity along the z-axis is a manifestation of the scaled dipolar Hamiltonian

FIG. 5. a) Functional dependence of the scaling term $\kappa$ on the pulse position $\epsilon$ in the various REDOR schemes. b) Functional dependence of the scaling term $\kappa_{\phi}$ on $\phi$. c) Functional dependence of the term multiplying the recoupled $\omega_{\mathrm{D}}^{( \pm 2)}$ in $m \mathcal{R}_{\epsilon_{1}}^{0.5}$ (or $m \mathcal{R}_{\epsilon_{1}}^{1.0}$ ) on $\phi$. Numerically simulated dependence of scaling factor $\kappa$ of the discussed REDOR schemes on d) pulse position $\epsilon$ and e) pulse ratio $\phi$ obtained from simulations. f) $\Delta S / S_{0}$ profiles of REDOR $\left(m \mathcal{R}_{0.25}^{0.75}\right)$ for a constant coupling of $1000 \mathrm{~Hz}$ for three $\phi$ values. $\Delta S / S_{0}$ profiles of $\mathrm{g}$ ) $m \mathcal{R}_{\epsilon_{1}}^{1.0}$ (solid lines) and $m \mathcal{R}_{\epsilon_{1}}^{0.5}$ (dotted lines) and h) $m \mathcal{R}_{\epsilon}^{0.5+\epsilon}$ for a few representative $\epsilon$ values. i) Minimum $R F$ requirements for the various REDOR schemes as a function of scaling in terms of MAS frequency.

FIG. 6. Experimental CN REDOR data (circles) and fits (lines) on glycine sample at $\omega_{\mathrm{r}}$ of $20 \mathrm{kHz}$ for a) $m \mathcal{R}_{\epsilon_{1}}^{0.5}$ and b) $m \mathcal{R}_{\epsilon}^{0.5+\epsilon}$ for a few values of $\epsilon$. 
7. CN REDOR data (circles) and fits (lines) for experiments involving $1 \mathrm{~ms}$ frequencyselective $\pi$ pulse on either $\mathrm{C}, \mathrm{N}$ or both channels for glycine sample at $\omega_{\mathrm{r}}$ of $20 \mathrm{kHz}$ and hard pulse data (plus) with fits (lines). a) $m \mathcal{R}_{0.085}^{0.585}$ with selective $\pi$ pulse on C, b) $m \mathcal{R}_{0.085}^{0.585}$ with selective $\pi$ pulse on $\mathrm{N}$, c) $m \mathcal{R}_{0.085}^{0.585}$, and d) $m \mathcal{R}_{0.05}^{0.55}$ with selective $\pi$ pulse on both $\mathrm{C}$ and $\mathrm{N}$ channels.

FIG. 8. Experimental $\Delta S / S_{0}$ profiles (circles) and fits (lines) on $\mathrm{C}_{\alpha}-\mathrm{H}_{\alpha}$ of deuterated alanine at $\omega_{\mathrm{r}}$ of $62.5 \mathrm{kHz}$ for a) $m \mathcal{R}_{0.25}^{0.75}(\mathrm{REDOR})$, b) $m \mathcal{R}_{0.25}^{0.5}$, c) $m \mathcal{R}_{0.2}^{0.5}$, d) $m \mathcal{R}_{0.1}^{0.5}$, e) $m \mathcal{R}_{0.125}^{0.625}$, f) $m \mathcal{R}_{0.085}^{0.585}$, g) $m \mathcal{R}_{0.05}^{0.55}$ and h) $m \mathcal{R}_{0.02}^{0.52}$.

TABLE I. Experimentally estimated dipolar couplings of $2-{ }^{13} \mathrm{C},{ }^{15} \mathrm{~N}$-Glycine at MAS frequency of $20 \mathrm{kHz}$

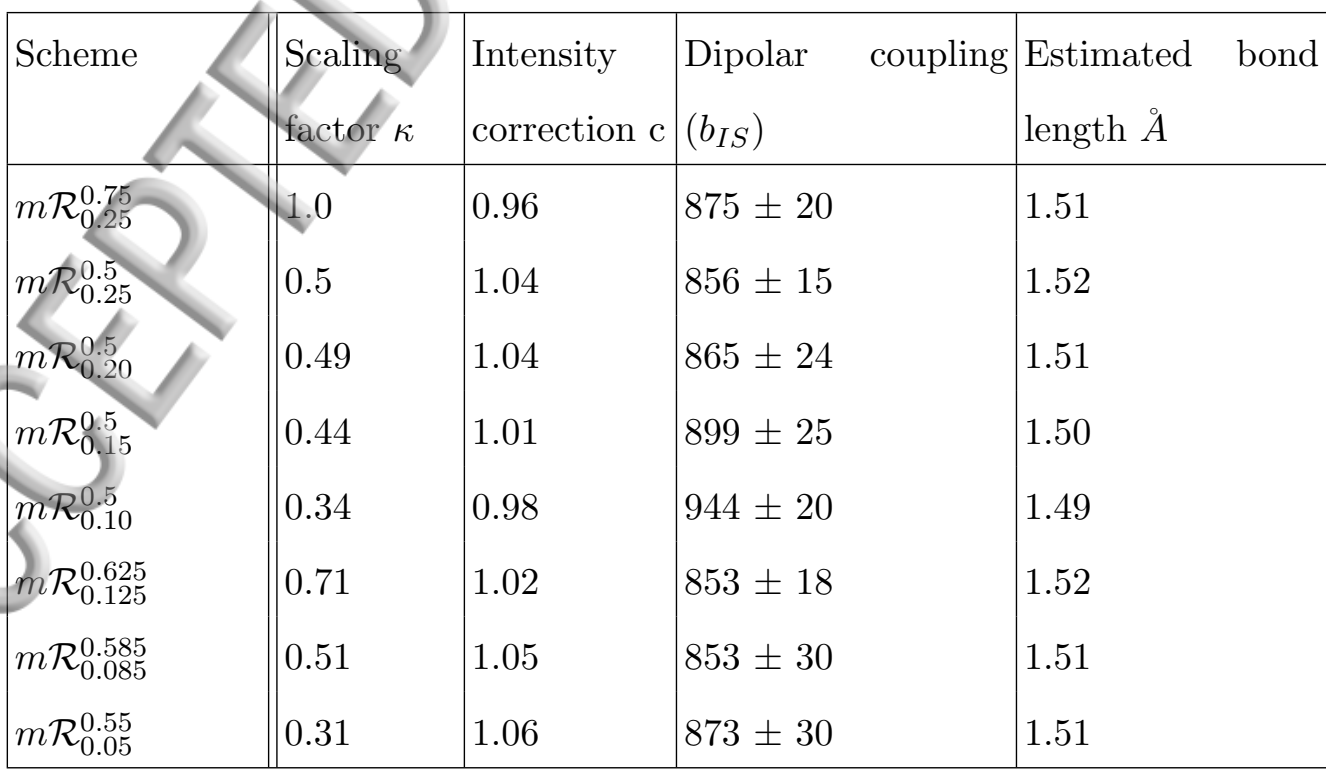




\section{Publishi.RG FERENCES}

${ }^{1}$ T. Gullion, J. Schaefer, Rotational-echo double-resonance NMR, J. Magn. Reson. 81 (1989) 196-200.

${ }^{2}$ M. Hong, Oligomeric structure, dynamics and orientation of mémbrane proteins from solid-state NMR, Structure, 14 (2006) 1731-1740.

${ }^{3}$ S. Dusold, A. Sebald, Dipolar recoupling under magic-angle spinning conditions, Annu. Rep. NMR Spectrosc. 41 (2000) 185-264.

${ }^{4}$ M. E. Merrit, J. M. Goetz, D. Whitney, C. P. Paul, L. Heux, J. L. Halary, J. Schaefer, Location of the antiplasticizer in cross-linked Epoxy resins by ${ }^{2} \mathrm{H},{ }^{15} \mathrm{~N}$ and ${ }^{13} \mathrm{C}$ REDOR NMR, Macromolecules, 31 (1998) 1214.

${ }^{5}$ B. Chandra, D. Bhowmik, B. K. Maity, K. R. Mote, D. Dhara, R. Venkatramani, S. Maiti, P. K. Madhu, Major reaction coordinates linking transient Amyloid- $\beta$ oligomers to fibrils measured at atomic level, Biophys. J. 113 (2017) 805-816.

${ }^{6}$ J. C. C. Chan, N. A. Oyler, Wai-Ming Yau, R. Tycko, Parallel $\beta$-sheets and polar zippers in amyloid fibrils formed by residues 10-39 of the yeast prion protein ure2p, Biochemistry, 44 (2005) 10669-10680.

${ }^{7}$ B. Chandra, V. S. Mithu, D. Bhowmik, A. K. Das, B. Sahoo, S. Maiti, P. K. Madhu, Curcumin dictates divergent fates for the central salt bridges in Amyloid- $\beta_{40}$ and Amyloid$\beta_{42}$, Biophys. J. 112 (2017) 1597-1608.

${ }^{8}$ D. R. Studelska, L. M. Mcdowell, M. P. Espe, C. A. Klug, J. Schaefer, Slowed enzymatic turnover allows characterization of intermediates by solid-state NMR, Biochemistry, 36 (1997) 15555-15560.

${ }^{9}$ N. Sinha, K. Schmidt-Rohr, M. Hong, Compensation for pulse imperfections in rotationalecho double-resonance NMR by composite pulses and EXORCYCLE, J. Magn. Reson. 168 (2004) $358-365$.

${ }^{10}$ M. G. Jain, G. Rajalakshmi, A. Equbal, K. R. Mote, V. Agarwal, P. K. Madhu, SineSquared shifted pulses for recoupling interactions in Solid-State NMR, J. Chem. Phys. 146 (2017) 244201.

${ }^{11}$ T. Gullion, J. Schaefer, Detection of weak heteronuclear dipolar coupling by rotationalecho double-resonance nuclear magnetic resonance, Adv. Magn. Reson. 13 (1989) 57-83. 
Publishîig Schanda, B. H. Meier, M. Ernst, Accurate measurement of one-bond H-X heteronuclear dipolar couplings in MAS solid-state NMR, J. Magn. Reson. 210 (2011) 246-259.

${ }^{13}$ T. Gullion, Rotational-Echo, Double-Resonance NMR, in Modern Magnetic Resonance, edited by G. A. Webb (Springer, Dordrecht, 2008).

${ }^{14}$ J. C. Chan, H. Eckert, Dipolar coupling information in multispin systems: Application of a compensated REDOR NMR approach to inorganic phosphates, J. Magn. Reson. 147 (2000) 170-178.

${ }^{15}$ J. Ren, H. Eckert, A homonuclear rotational echo double-resonance method for measuring site-resolved distance distributions in $\mathrm{I}=1 / 2$ spin pairs, clusters, and multispin systems, Angew. Chem. Int. Ed. 51 (2012) 12888-12891.

${ }^{16}$ E. O. Stejskal, J. Schaefer, J. S. Waugh, Magic-angle spinning and polarization transfer in proton-enhanced NMR, J. Magn. Reson. 28, (1977) 105-112.

${ }^{17}$ M. M. Maricq, J. S. Waugh, NMR in rotating solids, J. Chem. Phys. 70 (1979) 3300-3316.

${ }^{18}$ M. Mehring, Principles of high resolution NMR in solids (Springer, Berlin, 1983).

${ }^{19}$ J. S. Waugh, Average Hamiltonian theory, In: D. M. Grant, R. K. Harris, eds. Encyclopedia of Nuclear Magnetic Resonance, vol.2. Chichester, UK: Wiley; (1996) 849-854.

${ }^{20} \mathrm{~A}$. Brinkmann, Introduction to average Hamiltonian theory. I. Basics, Concepts Magn. Reson. Part A, 45A (2016) e21414.

${ }^{21}$ T. Gullion, D. B. Baker, M. S. Conradi, New, compensated Carr-Purcell sequences, J. Magn. Reson. 89 (1990) 479-484.

${ }^{22}$ C. P. Jaroniec, B. A. Tounge, C. M. Rienstra, J. Herzfeld, R. G. Griffin, Recoupling of heteronuclear dipolar interactions with rotational-echo double-resonance at high magicangle spinning frequencies, J. Magn. Reson. 146 (2000) 132-139.

${ }^{23}$ C. P. Jaroniec, Dipolar recoupling: Heteronuclear, in Encyclopedia of NMR, edited by D. M. Grant and R. K. Harris (Wiley, chichester, 2002).

${ }^{24}$ M. Bak, J. T. Rasmussen, N. C. Nielsen, SIMPSON: A general simulation program for solid-state NMR spectroscopy, J. Magn. Reson. 147 (2000) 296-330.

257. Tosner, R. Andersen, B. Stevensson, M. Eden, N. C. Nielsen, T. Vosegaard, Computerintensive simulation of solid-state NMR experiments using SIMPSON, J. Magn. Reson. 246 (2014) 79-93.

${ }^{26}$ M. Bak, N. C. Nielsen, REPULSION, A novel approach to efficient powder averaging in solid-state NMR, J. Magn. Reson. 125 (1997) 132-139. 
Publishỉng Equbal, M. Bjerring, P. K. Madhu, N. C. Neilsen, Improving spectral resolution in biological solid-state NMR using supercycled rCW heteronuclear decoupling, Chem. Phys. Lett. 635 (2015) 339-344.

${ }^{28}$ A. Equbal, P. K. Madhu, B. H. Meier, N. C. Nielsen, M. Ernst, V. Agarwal, Parameter independent low-power heteronuclear decoupling for fast magic-angle spinning solid-state NMR, J. Chem. Phys. 146 (2017) 084202.

${ }^{29}$ F. Delaglio, S. Grzesiek, G. W. Vuister, G. Zhu, J. Pfeifer, A. Bax, NMRPipe: A multidimensional spectral processing system based on UNIX pipes, J. Biomol. NMR, 6 (1995) $277-293$. 
a) MAS

i)

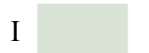

I

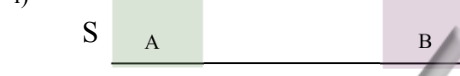

o.

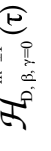
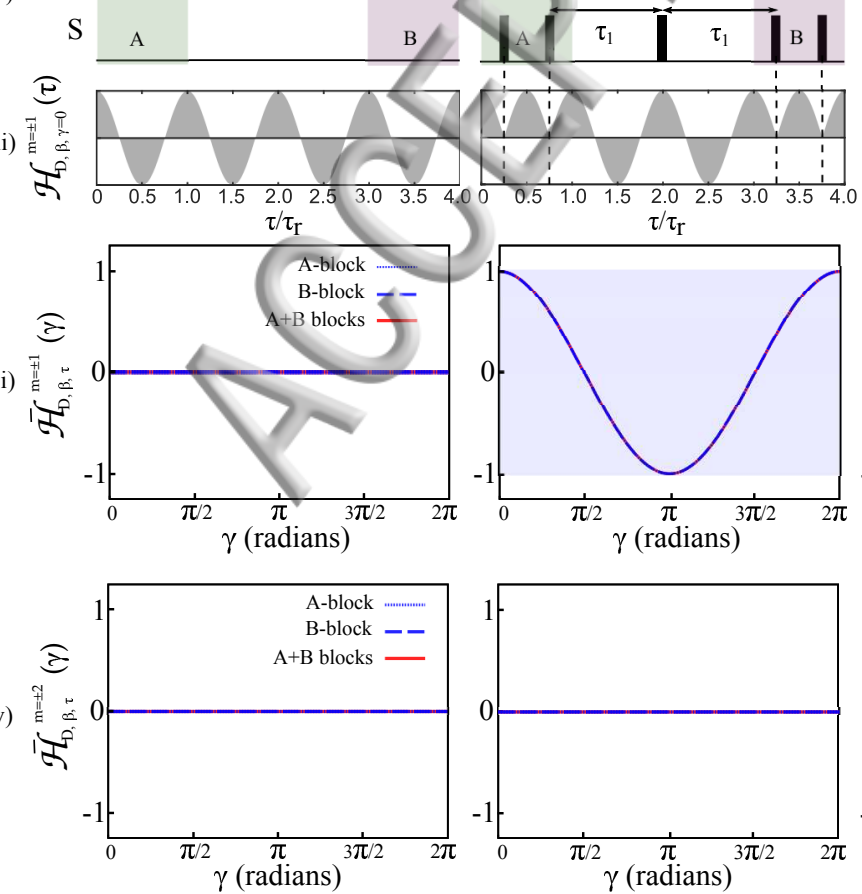
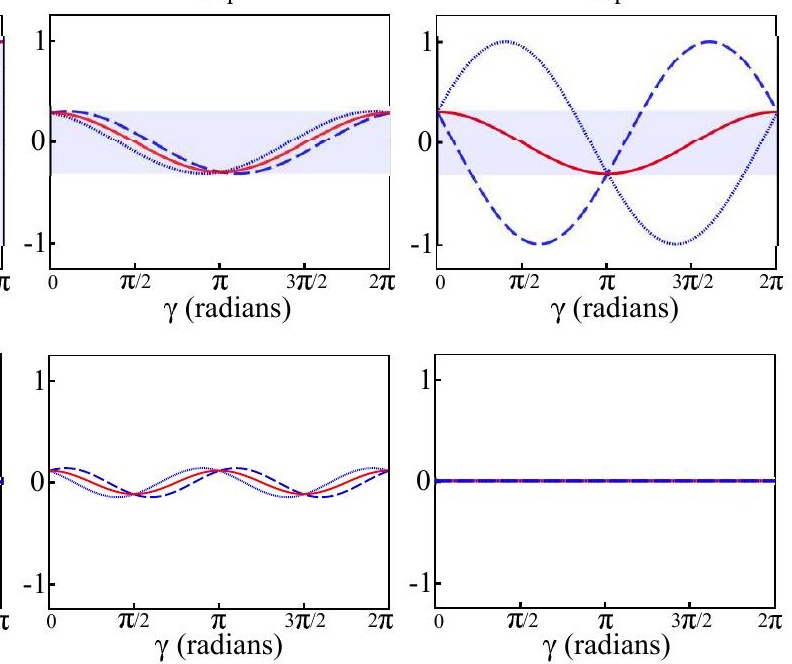


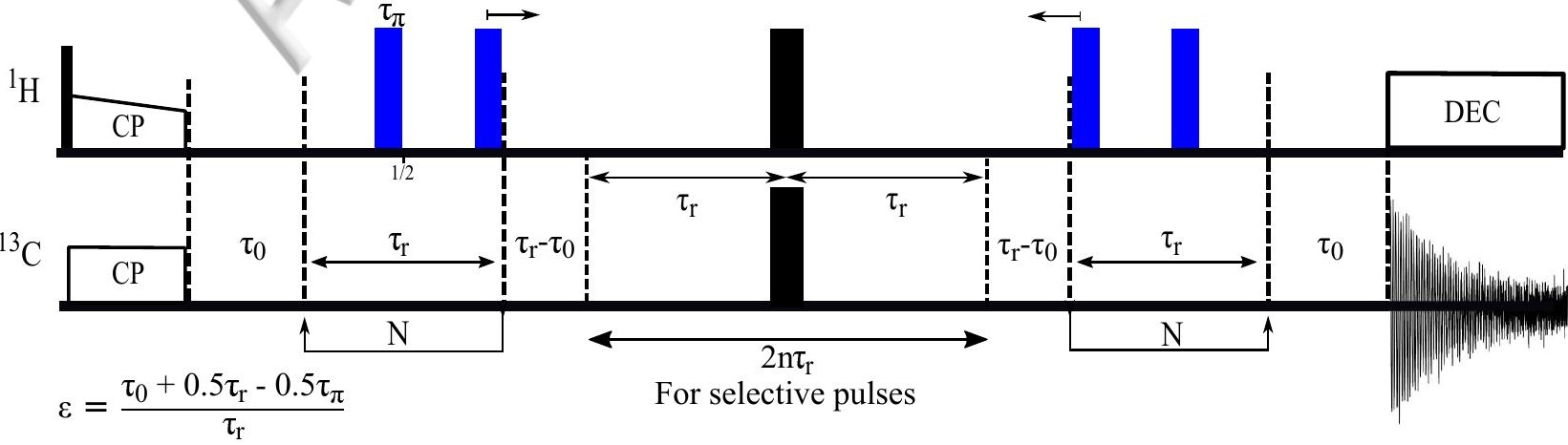



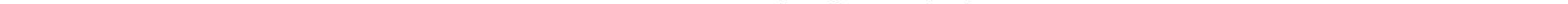

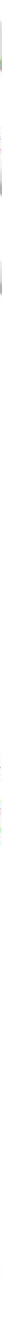


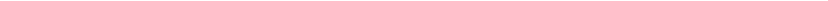




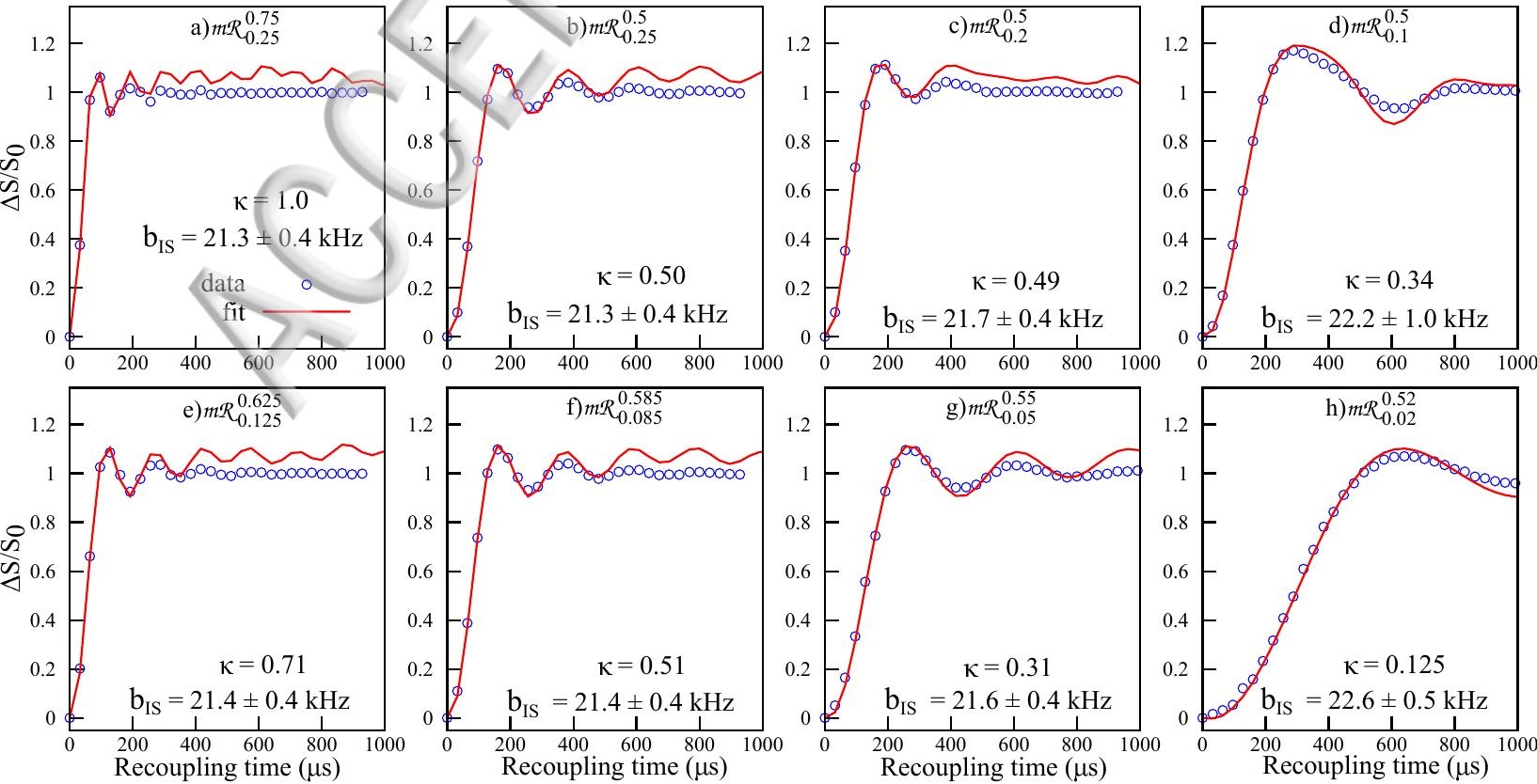

